\title{
Study of the likelihood of Alfvénic mode bifurcation in NSTX and predictions for ITER baseline scenarios
}

\author{
V. N. Duarte* \\ Princeton Plasma Physics Laboratory, Princeton University, Princeton, NJ, 08543, USA and \\ Institute of Physics, University of São Paulo, São Paulo, SP, 05508-090, Brazil \\ N. N. Gorelenkov, M. Schneller, E. D. Fredrickson, and M. Podestà \\ Princeton Plasma Physics Laboratory, Princeton University, Princeton, NJ, 08543, USA \\ H. L. Berk \\ Institute for Fusion Studies, University of Texas, Austin, TX, 78712, USA
}

(Dated: June 15, 2022)

\begin{abstract}
Rare Alfvénic wave transitions between fixed-frequency and chirping phases are identified in NSTX, where Alfvénic waves are normally observed to exhibit either chirping or avalanching responses. For those transitions, we apply a criterion [Duarte et al, Nucl. Fusion 57, 054001 (2017)] to predict the nature of fast ion redistribution in tokamaks to be in the convective or diffusive nonlinear regimes. For NSTX discharges in which the transition is not accompanied by changes in the beam deposited power or modifications in the injected radiofrequency power, it has been found that the anomalous fast ion transport is a likely mediator of the bifurcation between the fixed-frequency mode behavior and rapid chirping. For a quantitative assessment, global gyrokinetic simulations of the effects of electrostatic ion temperature gradient turbulence and trapped electron mode turbulence on chirping were pursued using the GTS code. The investigation is extended by means of predictive studies of the probable spectral behavior of Alfvénic eigenmodes for baseline ITER cases consisting of elmy, advanced and hybrid scenarios. It has been observed that most modes are found to be borderline between the steady and the chirping phases.
\end{abstract}

\section{INTRODUCTION}

Energetic-particle-driven Alfvénic instabilities can seriously degrade the performance of presentday and next-generation fusion devices [1-5]. The control of these instabilities is, therefore, considered essential for the ITER performance $[3,6]$. To avoid and mitigate instabilities due to fast ions, it is important to understand what is the nature of the induced transport (e.g., coherent prompt losses [7], convective losses due to phase-space structures [8] and diffusive losses due to phase-space stochastization [9]) and the associated spectral character of the instability [10]. For this purpose, in this paper we investigate what conditions delineate the transition of Alfvénic modes between fixed-frequency and chirping phases in NSTX. In addition to that, typical ITER cases are analyzed and predictions are

\footnotetext{
*vduarte@pppl.gov
}

made regarding the likelihood of modes to chirp or to oscillate steadily at a nearly constant frequency.

From the theory perspective, the onset of chirping has been linked with the relative importance between stochastic and coherent processes affecting the resonant population [11-14]. Experimentally, several elements have been identified as altering the Alfvén wave spectral behavior, such as radiofrequency (RF) waves [15-17], background plasma beta [18], beam beta [8], 3D fields [19] and rotational transform [20, 21]. Recently, the rare emergence of chirping in DIII-D has been shown to be related to a marked decrease of the inferred fast ion micro-turbulence levels [10].

In Refs. [10, 22], theoretical predictions for realistically computed tokamak modes were compared with experiments. It was observed, both in the theory and in the experiment, that fast ion microturbulence is a mediator between mode nature transition for several typical tokamak scenarios, while exhibiting little macroscopic anomalous transport 
[23]. The fact that ion anomalous diffusity is typically much smaller in spherical tokamaks (STs) than in conventional tokamaks has been proposed [10] as the explanation for the longstanding observation that chirping is more common in STs relative to conventional tokamaks. A criterion proposed to distinguish between the two typical scenarios (chirping vs fixed-frequency), which was shown to be sensitive to the relative strength of scattering (from collisions and micro-turbulence) and drag processes, ultimately translates into a condition for the applicability of reduced quasilinear modeling for realistic tokamak eigenmodes. Recently, DIII-D had dedicated experiments [24] to stress-test the chirping prediction [10]. The experiments employed negative plasma triangularity as a means to decrease turbulence levels. In those shots, chirping was much more prevalent than in the usual and more turbulent oval or positive triangularity cases. In addition, chirping was also observed for modes located around internal transport barriers, even in positive triangularity. From the numerical side, turbulence stochasticity has been recently included in a bump-on-tail simulation [25], which showed its effect on chirping suppression.

The purpose of this paper is twofold. First, we investigate whether the conclusion of chirping based on turbulence holds for NSTX, where ion microturbulence is already observed to be low (as compared to ion neoclassical transport [26]), or whether there are other elements that determine the mode nonlinear evolution. This is addressed by means of global gyrokinetic simulations. Chirping is a major issue in connection with fast ion losses in tokamaks and there is currently no understanding of how to systematically avoid them in NSTX-U. The second goal of this work is an attempt to anticipate what will be the probable spectral nature of toroidicity-induced and reversed shear Alfvén eigenmodes (TAEs and RSAEs) in ITER. The profiles employed in this study are fiducially constructed using a transport code.

This article is organized as follows. In Sec. II, we present gyrokinetic analysis of Alfvénic mode bifurcation in NSTX and its comparison with theoretical predictions. Sec. III is devoted to a study of the possibility of occurrence of mode chirping in ITER baseline scenarios (reversed shear, hybrid and elmy
$\mathrm{H}$ mode) and Sec. IV presents discussions and conclusions.

\section{ANALYSES OF RARE TRANSITIONS BETWEEN CONSTANT FREQUENCY AND CHIRPING IN NSTX}

A distinctive feature of Alfvénic wave behavior in STs [27] as compared to conventional tokamaks is that chirping and avalanches are customary in the former and infrequent in the latter. The ubiquitous Alfvénic chirping in NSTX is observed to be a precursor of the phase locking of high-intensity modes of several toroidal mode numbers, known as avalanche [28, 29]. During the avalanche, the escape of a substantial fraction of fast ions occurs, which can typically reach up to $40 \%$. Wave chirping has been identified to transition to avalanches in NSTX only when the fast particle energy was greater than about $30 \%$ of the total plasma energy [30], with higher chances of avalanches to appear correlating with lower values of maximum energetic ion speed divided by the Alfvén speed [27]. The conditions determining the likelihood of the chirpingto-avalanche transition are therefore, to a certain extent, experimentally identified. We therefore turn our attention to the likelihood of transition from a steady frequency to chirping instead.

Typically in NSTX, Afvénic modes are already chirping when they first appear in a spectrogram, although mode intensity can vary considerably. The constant frequency phase is normally not observed. Eventually chirping modes undergo conversion to the avalanche phase as more beam power is injected. It is often challenging to identify cases in which they appear in their fixed-frequency phase. From an extensive NSTX database, we have selected rare steady-to-chirping transitions, with no influence of $\mathrm{RF}$ or 3D fields, in order to test the proposed interpretation that fast ion micro-turbulence can be a determining factor behind the transition. Although rare, these transitions offer a unique testbed to decipher the parameters that need to be varied to induce change in the nonlinear character of modes. For some cases, we have observed that chirping and steady phases appear to co-exist for different Alfvénic modes, which possibly indicate that each 
mode has its own threshold to undergo transition between the two phases. From the initial set of transitions, we observe that in most cases the modes switch over to chirping because of an increase in the applied beam power, with the spectral change happening within the characteristic fast ion slowing down time. This increases the strength of the drive and likely allows the modes to access a harder nonlinear phase. This observation is in agreement with Ref. [8], that reported a correlation between chirping and high fast ion pressure. The chances of chirping increasing at higher drive may be related to a convective amplification mechanism [31] and also be related to mode structure deformation due to the presence of EPs, both of which are beyond the scope of this work. Therefore, to obtain a comparable set of steady-to-chirping transitions, we further analyze only a sub-set of cases with roughly constant beam beta throughout the transition. Only three discharges satisfied this constraint, among which only two allowed for a successful equilibrium reconstruction: NSTX shots \#128453 and \#135388.
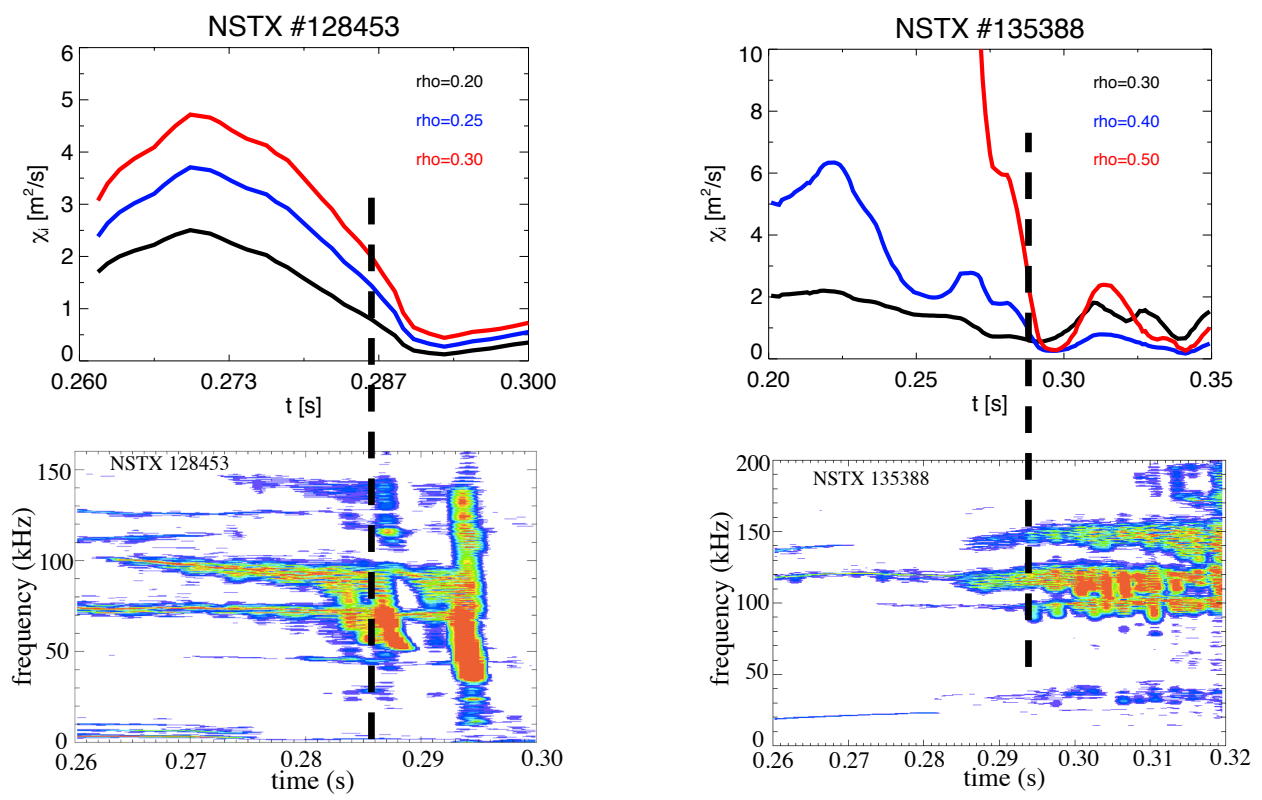

Figure 1. Correlation between the onset of Alfvénic chirping (lower plots) and an improvement of thermal ion confinement, as inferred from TRANSP (upper plots), for NSTX pulses \#128453 and \#135388.

Previous chirping analysis [10, 22] relied on transport coefficients calculated by TRANSP for thermal ions. The fast ion coefficients were then scaled from the thermal ones using the relations reported in Ref. [32-34]. These are based on an analytic approach and numerical examination of a simulation database. One can appreciate in Fig. 1 the correlation between the emergence of chirping and the decrease of thermal ion turbulent levels, which can be used as a proxy for the fast ion anomalous diffusivity [35]. The values of $\chi_{i}$ in Fig. 1 are unusually high before the start of the chirps. In the present work, however, we analyze new cases more quantitatively consistent by performing individual gyrokinetic simulations at given time slices of the mode evolution, taking into consideration the ex- 
perimental plasma parameters for each individual discharge. This allows us to understand what type of turbulence is dominant and offers insights on its key characteristics, such as the origin of its drive.

The global turbulence simulations reported in this study are carried out using the GYROKINETIC Tokamak Simulation (GTS) code [36, 37]. The GTS code performs nonlinear gradient-driven electrostatic turbulence simulations based on a generalized gyrokinetic simulation model using a $\delta f$ particle-in-cell approach. The presented GTS simulations of NSTX discharges \#128453 and \#135388 take into account a comprehensive influence of a number of relevant physical effects, including fully kinetic electrons, realistic geometry constructed using experimental data as well as plasma profiles which are read from TRANSP [38]. The global simulations cover a wide region of normalized minor radii, from $r_{\text {tor }}=0.2$ to $0.8\left(r_{\text {tor }}\right.$ represents the square root of the toroidal flux normalized with its value at the separatrix). Convergence in marker numbers was found for 80 particles per cell per species. The spatial grid size in the perpendicular direction is approximately equal to or less than the local ion gyroradius $\rho_{\mathrm{i}}$. The wavenumber range that is simulated is $k_{\perp} \rho_{\mathrm{i}} \lesssim 2$, which covers the typical low- $k$ turbulence due to ion temperature gradient (ITG) mode and trapped electron mode (TEM).

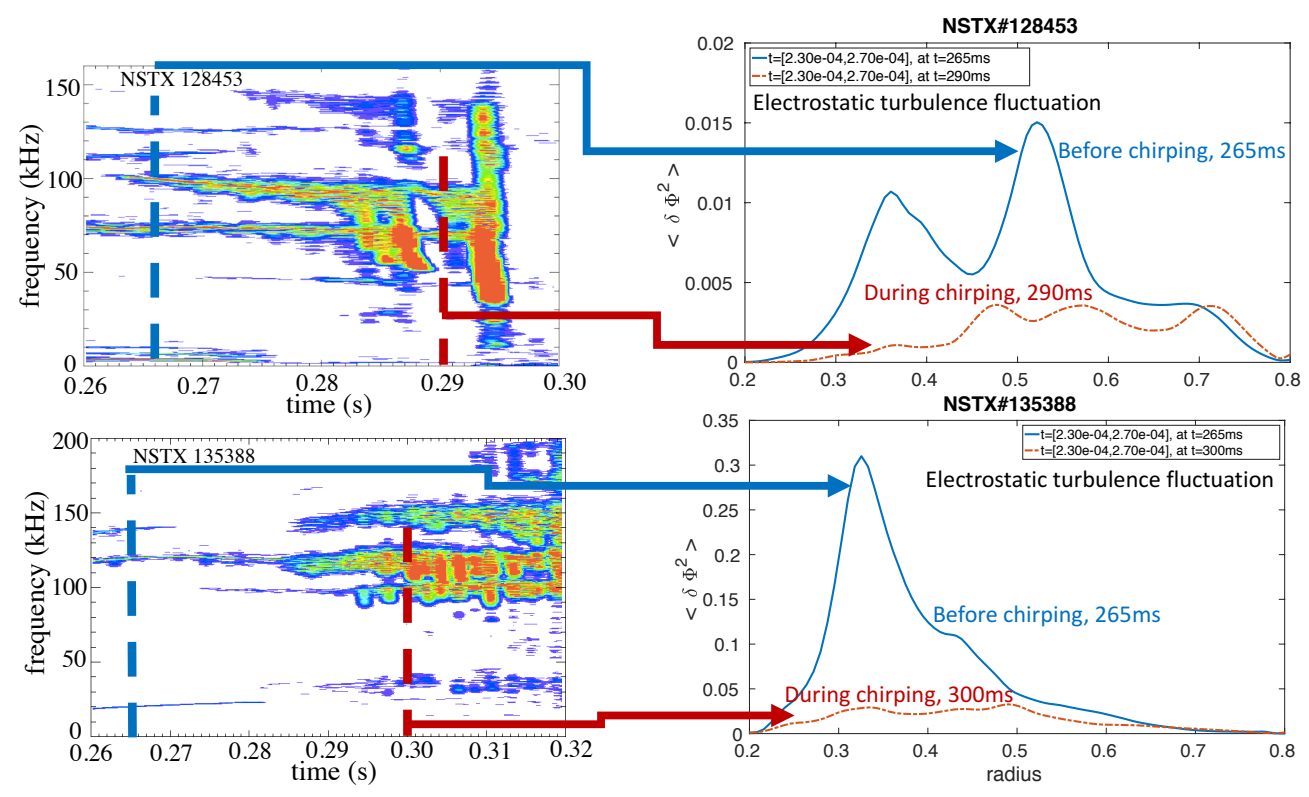

Figure 2. Correlation between low anomalous transport and Alfvénic chirping, as calculated by GTS global gyrokinetic simulations, for NSTX pulses \#128453 and \#135388.

The spatiotemporal evolution of turbulence intensity, defined as $\left\langle\delta \Phi^{2}\right\rangle \equiv\left\langle\left(e \delta \phi / T_{\mathrm{i}}\right)^{2}\right\rangle$, with $\delta \phi$ being the electrostatic potential fluctuation, $T_{\mathrm{i}}$ the ion temperature at the reference radius $r_{t o r}=0.5$ and $e$ the elementary charge, is displayed in Figs. 2, 3 and 4. For \#128453, the fully developed ITG/TEM turbulence spreads mainly around $r_{\text {tor }}=0.36$ and $r_{\text {tor }}=0.52$. For \#135388, ITG turbulence evolves between $r_{\text {tor }}=0.25$ and 0.6 with a peak around $r_{\text {tor }}=0.32$. The location of the peaks is consistent with the radial position with strongest temperature profile gradients. Interestingly, for both 
cases, the level of turbulence is remarkably reduced as the mode undergoes transition to chirping, in agreement with the theory prediction and the observation on DIII-D [10]. The evolution and saturation of the potential perturbation are illustrated in logarithmic scale in Fig. 5, using the equilibrium profiles before and during the chirping.

Interestingly, the turbulent levels in NSTX discharges \#128453 and \#135388 are found to be reduced due to different effects. For \#128453, it is observed (Fig. 6(a))that the ion temperature gets increasingly flattened in the core between $t=265 \mathrm{~ms}$ and $t=300 \mathrm{~ms}$, thus depleting the drive of the ITG modes. For \#135388, the $q$ profile, which was monotonic at $t=265 \mathrm{~ms}$, becomes reversed at $t=290 \mathrm{~ms}$ (Fig. 6(b)). The simulation result is in agreement with the prediction that a reversed $q$ profile should have an effect on the micro-turbulence supression [39].

The extraordinarily high electrostatic turbulence potential for case \#135388 (as compared to \#128453) can be partially understood when looking at one of the main driving sources, $\eta=\nabla T_{\mathrm{i}} / \nabla n_{\mathrm{i}}$. In both time slices of the discharge NSTX \#135388, this value is up to a factor of 5 to 6 higher than in the scenarios of NSTX \#128453. Especially at lower radii (where the stabilizing shear is lower), this high $\eta$ value is considered responsible for the high turbulence levels.

In order to evaluate the criterion for chirping likelihood proposed in [10], we first need to categorize the modes that are measured. For this purpose, we employ the reflectometer data to provide information regarding the mode structure, which can be compared with the eigenmodes calculated by the NOVA code [40, 41]. Due to lower density, for shot \#135388 only two channels of the reflectometer could be used, which did not allow for proper mode identification. The measured mode structure compared to its best match from NOVA results is shown in Fig. 7 for shot \#128453 before and during the chirping phase.

In order to calculate the relative importance between fast ion stochasticity arising from turbulent processes and collisional processes, we use the approach introduced by Lang and Fu [42], which in the notation defined in [22], is approximately given
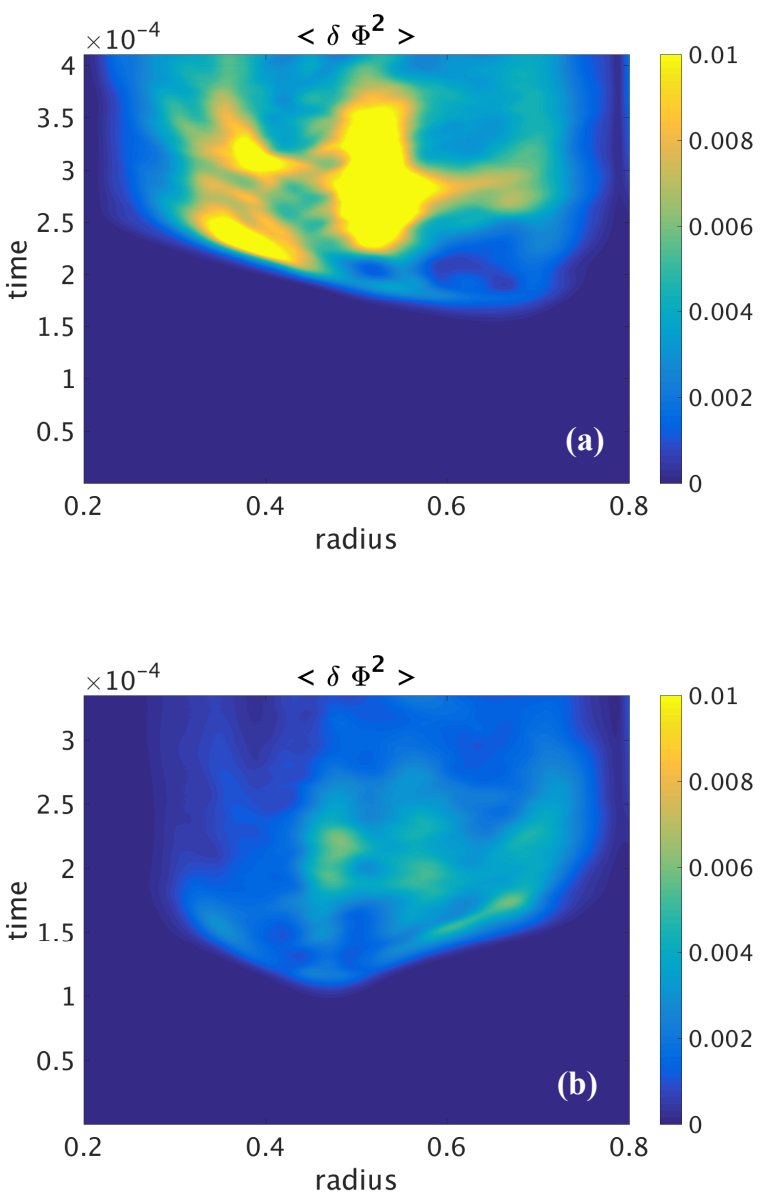

Figure 3. Averaged squared electrostatic potential fluctuation normalized with the square of the ion temperature at mid-radius (indicated by the color code), as a function of time, expressed in units of $10^{-4} \mathrm{~s}$ and the minor radius, expressed in terms of the square root of the toroidal flux divided by the toroidal flux at the edge. The results were obtained by the GTS code for the time slices (a) before chirping starts, at $t=265 \mathrm{~ms}$ and (b) during the chirping, at $t=290 \mathrm{~ms}$, for NSTX discharge \#128453. The color code scale is the same for both plots, which indicates a substantial reduction of the turbulent activity from (a) to (b). 

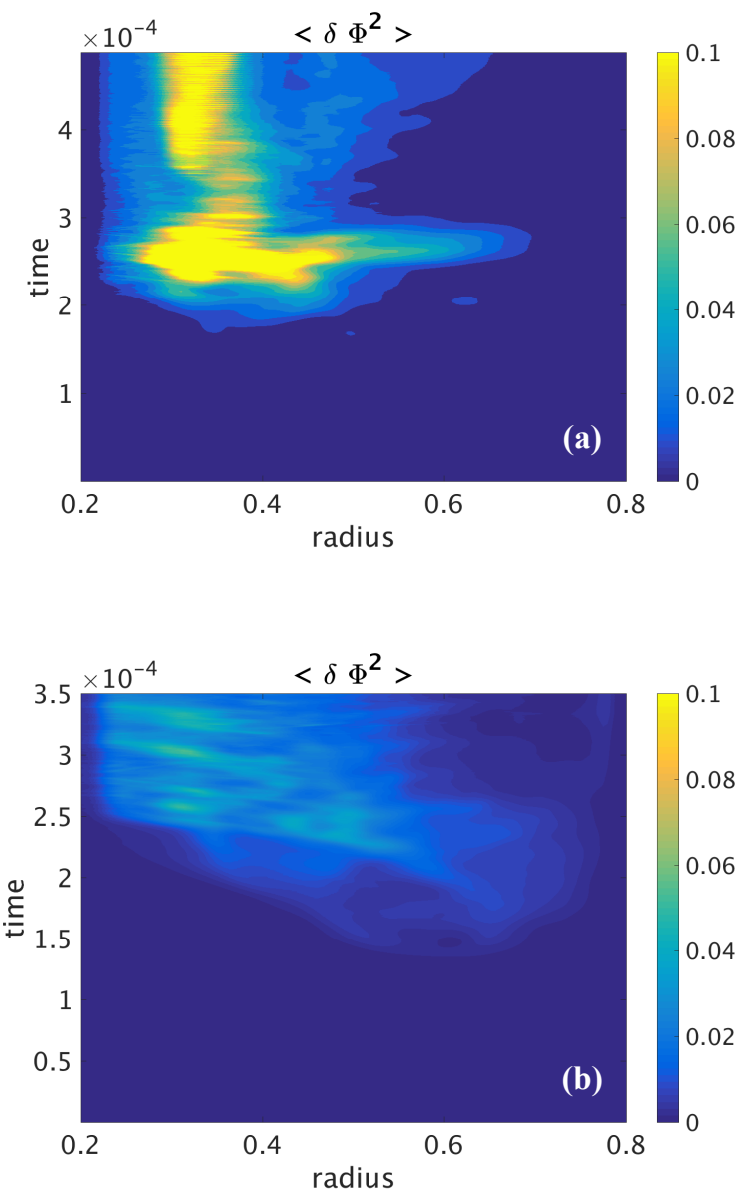

Figure 4. Averaged squared electrostatic potential fluctuation normalized with the square of the ion temperature at mid-radius (indicated by the color code), as a function of time, expressed in units of $10^{-4} s$ and the minor radius, expressed in terms of the square root of the toroidal flux divided by the toroidal flux at the edge. The results were obtained by the GTS code for the time slices (a) before chirping starts, at $t=265 \mathrm{~ms}$ and (b) during the chirping, at $t=300 \mathrm{~ms}$, for NSTX discharge \#135388. The color code scale is the same for both plots, which indicates a substantial reduction of the turbulent activity from (a) to (b).
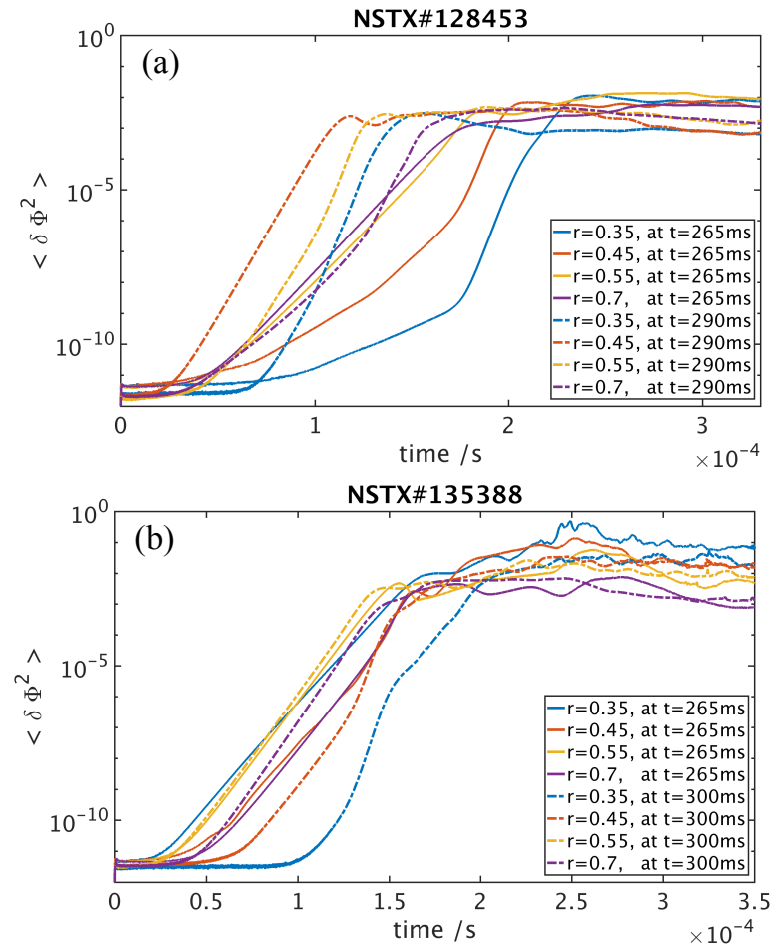

Figure 5. GTS computation of the time evolution of the averaged squared electrostatic potential fluctuation normalized with the square of the ion temperature at midradius, as a function of time for NSTX discharges (a) \#128453 and (b) \#135388, before (continuous curves) and during (dashed curves) the chirping. The colors indicate several radial positions.

by

$$
\frac{\nu_{\text {turb }}}{\nu_{\text {scatt }}} \simeq\left[\frac{D_{E P}\left(\frac{q_{E P}}{m_{E P}} \frac{\partial \psi}{\partial r}\right)^{2}}{2 \nu_{\perp} R^{2} \mu B}\right]^{1 / 3}
$$

where $\nu_{\text {turb }}$ and $\nu_{\text {scatt }}$ are the effective turbulent and collisional scattering frequencies, $D_{E P}, q_{E P}$ and $m_{E P}$ are the energetic particle diffusivity, charge and mass, respectively. $\psi$ is the poloidal magnetic flux divided by $2 \pi, r$ is the minor radius, $\nu_{\perp}$ is the $90^{\circ}$ pitch angle scattering rate, $R$ is the major radius, $\mu$ is the magnetic moment and $B$ is the magnetic field intensity. $D_{E P}$ is calculated using the scalings of [32]. We note, however, that the GTS 
code is currently being extended to include a passive energetic ion population. It should allow for more accurate estimates of $D_{E P}$ in the future.

The weakly nonlinear mode dynamics can be described by an integro-differential, time-delayed cubic equation for the mode amplitude [11, 43]. The evolution to chirping has been identified to be associated with the explosion of the solution of the cubic equation in a finite time [44]. It has been shown that, for realistic tokamak modes, a criterion for chirping likelihood Crt [10] should involve a phasespace integration over the multiple resonance surfaces of a given mode. Crt is sensitive to the ratio between the effective frequencies due to stochastic $\left(\nu_{\text {stoch }}\right)$ and coherent $\left(\nu_{\text {coher }}\right)$ processes. We take $\nu_{\text {stoch }}=\nu_{\text {scatt }}+\nu_{\text {turb }}$ and $\nu_{\text {coher }}=\nu_{\text {drag }}$, where $\nu_{d r a g}$ is the effective frequency associated with collisional drag (slowing down). For the chirping criterion $C r t$ evaluation purpose, we use the kinetic postprocessor NOVA-K [45, 46]. We find that at 265ms, Crt $=+0.0027$ and at $290 \mathrm{~ms}$, Crt $=-0.34$, which is consistent with the experiment $(C r t<0$ implies more probability of chirping while $C r t>0$ predicts that the wave will likely oscillate at a constant frequency [10]).

\section{PREDICTIVE STUDIES FOR ITER BASELINE SCENARIOS}

ITER will employ two negative-ion-based neutral beam injection (NBI) sources, which will account for $33 M W$ of injected power [47, 48]. Both the $3.5 \mathrm{MeV}$ fusion-born alpha particles and the tangentially injected $1 \mathrm{MeV}$ NBI ions will have supraAlfvénic velocities, allowing them to interact with TAEs via their main resonance. An upper limit of $5 \%$ of fast ion loss has been established for ITER to sustain a burning plasma [6]. Therefore, to understand the relevant mode evolution and fast ion transport due to Alfvénic instabilities in ITER, it is instructive to anticipate whether the modes will be more prone to have their frequencies locked to the background equilibrium or be subject to rapid chirps [49]. In recent years, a number of publications have addressed various aspects of fast ion confinement in ITER, both linearly and nonlinearly [50-62]. Most of these previous studies quantita-


Figure 6. Ion temperature profile (red) and safety factor profile (black), before (continuous curves) and after (dot-dashed curves) the transition to chirping. Simulations show that for shot 128453 (part (a)), the turbulence drive is depleted via a flattening of $T_{i}$ at midradius while for shot 135388 (part (b)), it is found that the reversal of $q$ has a stabilizing effect on the turbulence.

tively addressed stability and transport features. In this work we analyze another relevant aspect of the problem, which is the prediction of the probable character that Alfvénic waves will assume in ITER. This can be helpful in anticipating the theoretical and numerical tools that may need to be developed and employed. For example, for situations in which chirping is not expected to take place, re- 

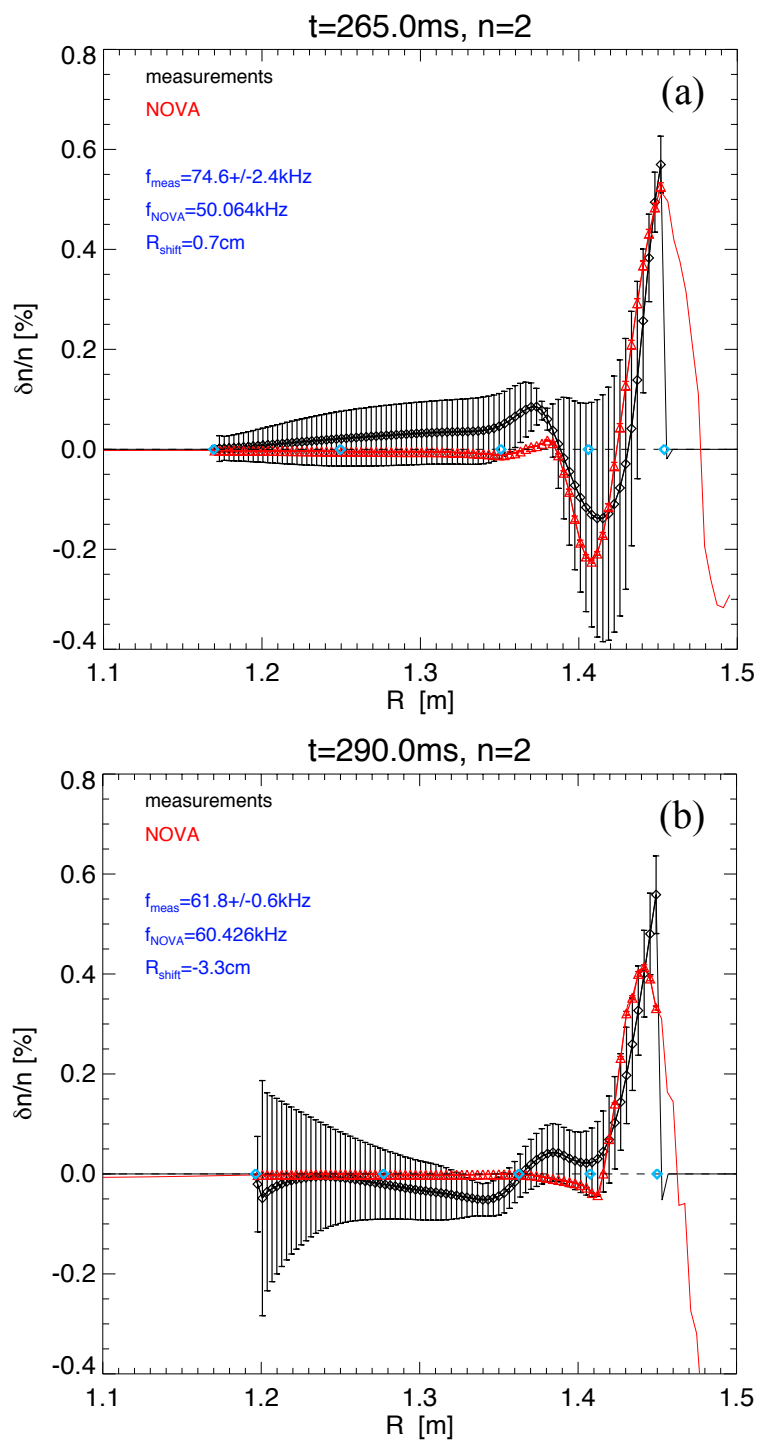

Figure 7. Identification of mode structures for an $n=2$ mode NSTX shot 128453 calculated by NOVA (in red) compared with reflectometer measurements (in black) for (a) $t=265 \mathrm{~ms}$ and (b) $t=290 \mathrm{~ms}$. $\delta n$ is the perturbed density while $n$ is the background density. The reflectometer measurement positions are shown by the cyan diamonds. duced quasilinear modeling [63-65] can be enough for a quantitative assessment of fast ion redistribution due to Alfvénic modes.

ITER plasma performance can change considerably depending on the relative external heating power between NBI, ion cyclotron resonant heating $(\mathrm{ICRH})$ and electron cyclotron resonant heating $(\mathrm{ECRH})$. Heating mixes with high NBI power increase the toroidal rotation and the fusion yield but have the disadvantage of triggering Alfvénic instabilities [66]. Therefore it is necessary to consider Alfvénic spectral behavior for the main three scenarios [67] and make use of different plasma profiles that the TRANSP code predicts for them. The first scenario is a reversed shear, advanced (steady state) scenario in which most of the plasma current is driven non-inductively (bootstrap current), with the current density peak displaced from the center and a non-monotonic safety factor $q$ profile. The second scenario is an elmy $\mathrm{H}$ mode, in which $q$ is minimum (with a value just below 1 ) at the center. Finally, the third scenario is a hybrid one, that has weak or low magnetic shear and the current, although modified externally, does not completely rely on non-inductive mechanisms.

We utilize plasma parameters from a previous TRANSP/TSC analysis [57], requiring $Q \geq 10$ (where $Q$ is the ratio between the power generated by fusion reactions and the power input to the plasma). We employ the mode structures and resonances previously reported in [57], which had their linear stability already assessed. For each ITER scenario, we take the most linearly unstable modes and limit ourselves to toroidal mode numbers $n$ from 7 to 11 .

In order to be able to account for distinctive turbulence levels for each of the three baseline scenarios, we use the results of Ref. [32] to scale the fast ion diffusivity with the thermal ion one, which is obtained with TRANSP. We note that Albergante et al [68-70] used the GENE code [71] to study the diffusivity of fast ions due to ITG turbulence specifically for ITER-relevant scenarios [6, 67]. An alternative for our study would be to use the computed fast ion diffusivity in terms of the parallel and perpendicular components of the velocity, shown in Fig. 6 of Ref. [68]. We note that the figure was produced for the specific case of $E=30 T_{e}$. However, 


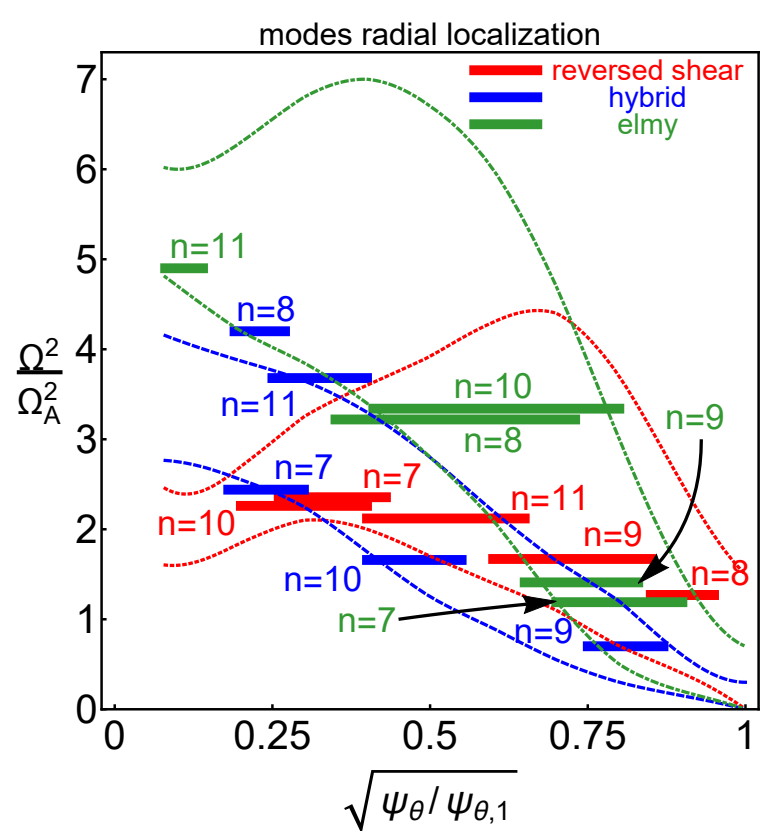

Figure 8. Radial localization and frequency of the TAEs and RSAEs used in the chirping prediction simulations of Fig. 9. Dotted (red), dashed (blue) and dot-dashed (green) curves indicate the envelope of the continuum for reversed-shear, hybrid and $\mathrm{H}$ mode elmy cases. For the purpose of obtaining the envelope, a high toroidal mode number $n=30$ was used. $\Psi_{\theta}$ is the poloidal magnetic flux and $\Psi_{\theta, 1}$ is its value at the plasma separatrix. The modes angular frequency $\Omega$ is nomalized with $\Omega_{A}=v_{A, a x i s} /\left(q_{1} R_{0}\right)$, where $v_{A, a x i s}$ is the Alfvén speed at the magnetic axis, $q_{1}$ is the safety factor at the edge and $R_{0}$ is the major radius at the geometric center.

to make an estimate less approximate, one can use the result in Fig. 5b of Ref. [68] that shows the dependence of the diffusivity on the temperature, for the situation when the velocity-space is integrated over, where it can be inferred that $D_{E P} \propto T_{i}^{-1}$. The latter expression can be used as a correction factor to the diffusivity resolved in velocity for the case $E=30 T_{e}$, in order to have a better estimate for the specific fast ion energy and background temperature for each specific ITER case. Interestingly, we find that the scalings found in Ref. [32] provide values for $D_{E P}$ reasonably compatible with the ones extracted from Albergante's work, for most cases examined here.
ITER reversed shear (advanced) scenario
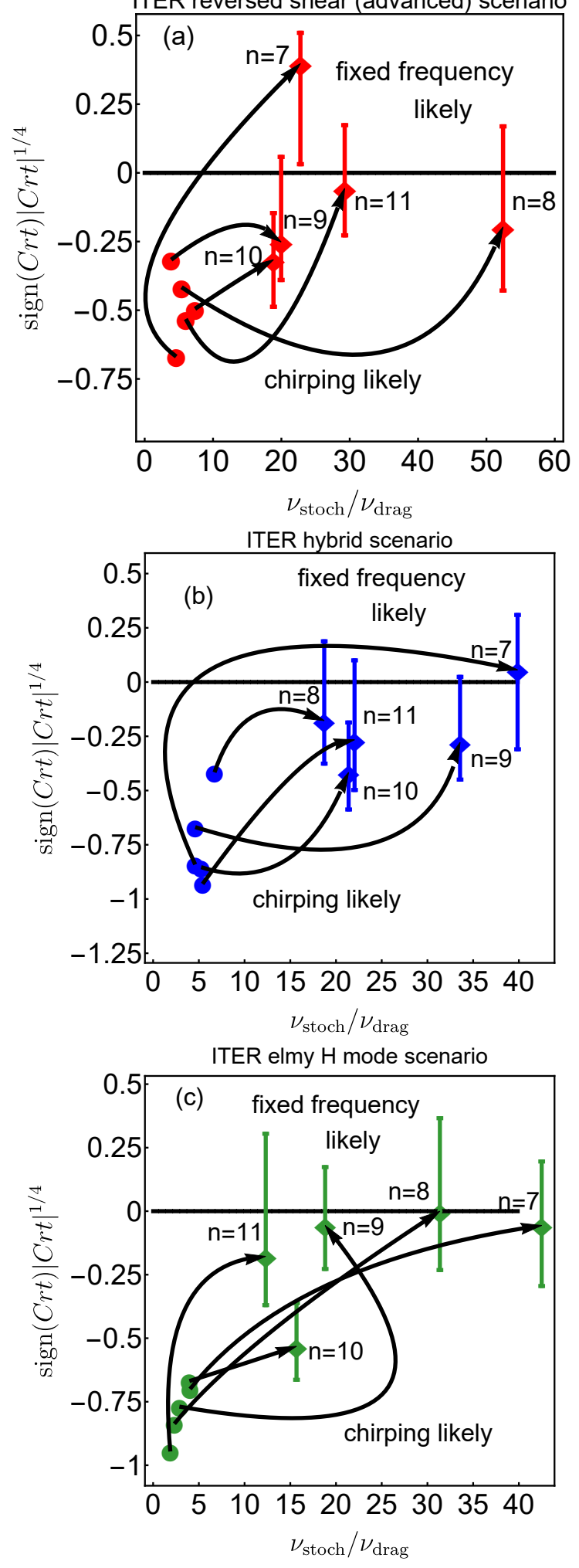

Figure 9. Evaluation of the chirping criterion Crt [10, 22] for the baseline scenarios (a) reversed shear, (b) hybrid and (c) elmy h mode. It is shown the prediction for individual modes with (arrowhead diamonds) and without (arrow tail disks) the inclusion of microturbulence stochasticity in the model. The arrows do not describe any meaningful path but simply connect two points corresponding to the same mode. 
The frequency and radial localization of the 15 modes used in the analysis are shown in Fig. 8, overlaid with the envelopes of the continua. These envelopes were calculated by interpolating the tips of each continuum curve, using high values of $n$ in order to be able to produce more reliable curves, representative of all modes of a given scenario.

The fact that the TAEs and the RSAEs in ITER can be driven by both alphas and beam ions is taken into account in the analysis via a weighted average of the individual contributions entering Eq. 1. The predictions for the three baseline scenarios for ITER are shown in Fig. 9. They indicate that most of the unstable TAEs and RSAEs are located close to the boundary between the chirping and steady frequency regions. We note that without the addition of micro-turbulent stochasticity in the model, all modes are predicted to lie in the region that allows for chirping. However, upon the addition of $\nu_{\text {turb }}$ to $\nu_{\text {scatt }}$ (represented by the arrows in Fig. 9), most modes get very close to the borderline $C r t=0$. Since there could be a considerable error in the estimate of $D_{E P}$, we show error bars in Fig. 9, which indicate by how much the prediction for Crt would change if $D_{E P}$ is multiplied by two (upper bars) and divided by two (lower bars).

It is worth comparing the predictions for ITER with previous analyses of DIII-D discharges [10]. Because of the inverse dependence of $D_{E P}$ on the EP energy, we see that for ITER, $D_{E P}$ is about an order of magnitude smaller with respect to typical values inferred for DIII-D. On the other hand, the Alfvén speed $v_{A}$ is about 2.5 times larger in ITER because of higher field, which means that the $90^{\circ}$ degree pitch angle scattering rate $\nu_{\perp}$ (see definition in Eq. 3 of Ref. [22]) at $v_{A}$ is also an order of magnitude smaller than in DIII-D. The combination of the other parameters involved in the ratio 1 do not change appreciably in the comparison between the two tokamaks, therefore, the ratio 1 appears to be of the same order in DIII-D and in ITER.

Each ITER mode in Fig. 9 appears to have its own threshold for steady/chirping transition. Therefore it is challenging to draw general conclusions regarding their spectral nature. It is however notorious that most modes appear to be close to the borderline. This means that any additional stochastic mechanism, such as RF heating, interaction with neoclassical tearing modes (NTMs), field ripples, energy diffusion, mode overlap, would contribute to make the modes cross towards the positive $\mathrm{Crt}$ region. ITER will be able to operate on a range of ICRH power, which can deliver up to $20 M W$ [66]. It remains to be understood how strong the effect of ICRH can be on the fast ions that are in resonance with Alfvén waves.

\section{SUMMARY AND CONCLUSIONS}

We reported a study of rare transitions between mode constant frequency and mode chirping in NSTX using global gyrokinetic simulation and chirping criterion analysis. The results indicate that fast ion anomalous diffusion likely mediates the transition, for cases when RF-induced diffusion and changes in beam beta are not present. The chirping phase is observed to be achieved following a marked decrease in the turbulent field amplitude, which is consistent with the interpretation proposed in Ref. [10].

We have studied the likelihood of TAEs to exhibit chirping for typical ITER scenarios (reversed shear, elmy and hybrid). A few modes were found to be distinctly on either oscillation regime. However, most modes were found to be borderline between the two phases. If additional mechanisms for detuning fast ion from a resonance (e.g., induced by 3D fields, ICRH, NTMs, energy diffusion) will be important in ITER, then most modes can be expected to oscillate at a constant frequency. Each of these additional mechanisms, however, deserve a study on their own, which is beyond the scope of this work. We also note that the constraint $Q>10$ imposed by the analysis means that the thermal diffusion could be too optimistic, which would increase the likelihood for chirping. Besides, this constraint may mean that we are overestimating the drive of the modes.

The analysis we presented in both parts of this work assumed that the modes remain isolated throughtout their evolution. We note, however, that because the toroidal mode numbers of Alfvénic waves in ITER will be higher compared to presentday tokamaks, also higher poloidal mode numbers will be important. This can lead to an enhanced 
phase-space density of resonances (as an illustration, see Fig. 9 of [53]), which can make resonance overlap more likely to occur. In the region of overlap, resonant particles should move stochastically, which contributes to destroy phase-space structures that sustain coherent phenomena, such as chirping. In this case, our predictions with respect to the nonlinear character of Alfvénic modes, which assumed isolated resonances, are no longer valid. In the future, the description of the chirping structures using more sophisticated numerical tools, such as guiding center codes or initial value codes, could provide a more detailed picture of the mode dynamics in realistic toroidal geometry. They could be used as a comparison with the predictions from the present reduced modeling effort.

\section{ACKNOWLEDGMENTS}

This work was supported by the US Department of Energy (DOE) under contract DE-AC02-
09CH11466 and by the São Paulo Research Foundation (FAPESP) under grants 2012/22830-2 and 2014/03289-4. The authors acknowledge Z. X. Lu for pointing out the stabilizing effect of a reversed shear profile, S. M. Kaye for preparing a TRANSP run for NSTX shot 128453 and S. Ethier for preparing the TRANSP profiles and equilibrium input files for GTS. The GTS simulations for this work were run on the supercomputer HYDRA, provided by the Max Planck Computing and Data Facility (MPCDF) in Garching, Germany. Computational resources as well as support are gratefully acknowledged.
[1] W. W. Heidbrink and G. J. Sadler, Nuclear Fusion 34, 535 (1994).

[2] P. Lauber, Physics Reports 533, 33 (2013).

[3] N. Gorelenkov, S. Pinches, and K. Toi, Nucl. Fusion 54, 125001 (2014).

[4] L. Chen and F. Zonca, Rev. Mod. Phys. 88, 015008 (2016).

[5] W. W. Heidbrink, Phys. Plasmas 15, 055501 (2008), http://dx.doi.org/10.1063/1.2838239.

[6] ITER Physics Expert Group on Energetic Particles, Heating and Current Drive and ITER Physics Basis Editors, Nuclear Fusion 39, 2471 (1999).

[7] X. Chen, M. E. Austin, R. K. Fisher, W. W. Heidbrink, G. J. Kramer, R. Nazikian, D. C. Pace, C. C. Petty, and M. A. Van Zeeland, Phys. Rev. Lett. 110, 065004 (2013).

[8] W. W. Heidbrink, Plasma Phys. Control. Fusion 37, 937 (1995).

[9] M. García-Muñoz, N. Hicks, R. van Voornveld, I. G. J. Classen, R. Bilato, V. Bobkov, M. Bruedgam, H.-U. Fahrbach, V. Igochine, S. Jaemsae, M. Maraschek, and K. Sassenberg (ASDEX Upgrade Team), Phys. Rev. Lett. 104, 185002 (2010).
[10] V. N. Duarte, H. L. Berk, N. N. Gorelenkov, W. W. Heidbrink, G. J. Kramer, R. Nazikian, D. C. Pace, M. Podestà , B. J. Tobias, and M. A. V. Zeeland, Nuclear Fusion 57, 054001 (2017).

[11] H. L. Berk, B. N. Breizman, and M. Pekker, Phys. Rev. Lett. 76, 1256 (1996).

[12] H. Berk, B. Breizman, and N. Petviashvili, Phys. Lett. A 234, 213 (1997).

[13] M. K. Lilley, B. N. Breizman, and S. E. Sharapov, Phys. Rev. Lett. 102 (2009), 10.1103/physrevlett.102.195003.

[14] M. K. Lilley, B. N. Breizman, and S. E. Sharapov, Phys. Plasmas 17, 092305 (2010), http://dx.doi.org/10.1063/1.3486535.

[15] D. Maslovsky, B. Levitt, and M. E. Mauel, Phys. Rev. Lett. 90, 185001 (2003).

[16] D. Maslovsky, B. Levitt, and M. E. Mauel, Physics of Plasmas 10, 1549 (2003), http://dx.doi.org/10.1063/1.1557072.

[17] E. Fredrickson, G. Taylor, N. Bertelli, D. Darrow, N. Gorelenkov, G. Kramer, D. Liu, N. Crocker, S. Kubota, and R. White, Nuclear Fusion 55, 013012 (2015).

[18] M. P. Gryaznevich and S. E. Sharapov, Plasma 
Physics and Controlled Fusion 46, S15 (2004).

[19] A. Bortolon, W. W. Heidbrink, G. J. Kramer, J.-K. Park, E. D. Fredrickson, J. D. Lore, and M. Podestà, Phys. Rev. Lett. 110, 265008 (2013).

[20] A. Melnikov, L. Eliseev, F. Castejón, C. Hidalgo, P. Khabanov, A. Kozachek, L. Krupnik, M. Liniers, S. Lysenko, J. de Pablos, S. Sharapov, M. Ufimtsev, V. Zenin, and HIBP Group and TJ-II Team, Nuclear Fusion 56, 112019 (2016).

[21] A. Melnikov, L. Eliseev, E. Ascasíbar, A. Cappa, F. Castejón, C. Hidalgo, T. Ido, J. Jiménez, A. Kozachek, L. Krupnik, M. Liniers, S. Lysenko, K. Nagaoka, J. de Pablos, A. Shimizu, S. Sharapov, M. Ufimtsev, S. Yamamoto, and HIBP group and TJ-II team, Nuclear Fusion 56, 076001 (2016).

[22] V. N. Duarte, H. L. Berk, N. N. Gorelenkov, W. W. Heidbrink, G. J. Kramer, R. Nazikian, D. C. Pace, M. Podestà, and M. A. V. Zeeland, Physics of Plasmas 24, 122508 (2017), https://doi.org/10.1063/1.5007811.

[23] D. C. Pace, M. E. Austin, E. M. Bass, R. V. Budny, W. W. Heidbrink, J. C. Hillesheim, C. T. Holcomb, M. Gorelenkova, B. A. Grierson, D. C. McCune, G. R. McKee, C. M. Muscatello, J. M. Park, C. C. Petty, T. L. Rhodes, G. M. Staebler, T. Suzuki, M. A. Van Zeeland, R. E. Waltz, G. Wang, A. E. White, Z. Yan, X. Yuan, and Y. B. Zhu, Phys. Plasmas 20, 056108 (2013).

[24] M. A. Van Zeeland et al (15th IAEA Technical Meeting on the Energetic Particles in Magnetic Confinement Systems, invited talk, 2017).

[25] B. J. Q. Woods, V. N. Duarte, A. J. De-Gol, N. N. Gorelenkov, and R. G. L. Vann, Nuclear Fusion (2018), 10.1088/1741-4326/aaa9fd.

[26] S. Kaye, F. Levinton, D. Stutman, K. Tritz, H. Yuh, M. Bell, R. Bell, C. Domier, D. Gates, W. Horton, J. Kim, B. LeBlanc, N. Luhmann, R. Maingi, E. Mazzucato, J. Menard, D. Mikkelsen, D. Mueller, H. Park, G. Rewoldt, S. Sabbagh, D. Smith, and W. Wang, Nucl. Fusion 47, 499 (2007).

[27] K. G. McClements and E. D. Fredrickson, Plasma Physics and Controlled Fusion 59, 053001 (2017).

[28] M. Podestà, R. Bell, A. Bortolon, N. Crocker, D. Darrow, A. Diallo, E. Fredrickson, G.-Y. Fu, N. Gorelenkov, W. Heidbrink, G. Kramer, S. Kubota, B. LeBlanc, S. Medley, and H. Yuh, Nucl. Fusion 52, 094001 (2012).

[29] E. D. Fredrickson, N. A. Crocker, R. E. Bell, D. S. Darrow, N. N. Gorelenkov, G. J. Kramer, S. Kubota, F. M. Levinton, D. Liu, S. S. Medley, M. Podestà, K. Tritz, R. B. White, and H. Yuh, Physics of Plasmas 16, 122505 (2009), https://doi.org/10.1063/1.3265965.

[30] E. Fredrickson, N. Gorelenkov, M. Podesta, A. Bortolon, S. Gerhardt, R. Bell, A. Diallo, and B. LeBlanc, Nuclear Fusion 54, 093007 (2014).

[31] F. Zonca, S. Briguglio, L. Chen, G. Fogaccia, and G. Vlad, Nuclear Fusion 45, 477 (2005).

[32] W. Zhang, Z. Lin, and L. Chen, Phys. Rev. Lett. 101 (2008), 10.1103/physrevlett.101.095001.

[33] T. Hauff, M. J. Pueschel, T. Dannert, and F. Jenko, Phys. Rev. Lett. 102, 075004 (2009).

[34] M. Pueschel, F. Jenko, M. Schneller, T. Hauff, S. Guenter, and G. Tardini, Nuclear Fusion 52, 103018 (2012).

[35] W. W. Heidbrink, J. M. Park, M. Murakami, C. C. Petty, C. Holcomb, and M. A. V. Zeeland, Phys. Rev. Lett. 103 (2009), 10.1103/physrevlett.103.175001.

[36] W. X. Wang, Z. Lin, W. M. Tang, W. W. Lee, S. Ethier, J. L. V. Lewandowski, G. Rewoldt, T. S. Hahm, and J. Manickam, Physics of Plasmas 13, 092505 (2006), http://dx.doi.org/10.1063/1.2338775.

[37] W. X. Wang, P. H. Diamond, T. S. Hahm, S. Ethier, G. Rewoldt, and W. M. Tang, Physics of Plasmas 17, 072511 (2010), http://dx.doi.org/10.1063/1.3459096.

[38] R. J. Hawryluk, in Physics of Plasmas Close to Thermonuclear Conditions, Vol. 1, edited by B. Coppi, G. G. Leotta, D. Pfirsch, R. Pozzoli, and E. Sindoni (CEC, Brussels, 1980) pp. 19-46.

[39] Y. Kishimoto, J.-Y. Kim, W. Horton, T. Tajima, M. J. LeBrun, and H. Shirai, Plasma Physics and Controlled Fusion 41, A663 (1999).

[40] C. Cheng, L. Chen, and M. Chance, Annals of Physics 161, 21 (1985).

[41] N. N. Gorelenkov, C. Z. Cheng, and G. Y. Fu, Phys. Plasmas 6, 2802 (1999).

[42] J. Lang and G.-Y. Fu, Phys. Plasmas 18, 055902 (2011).

[43] H. L. Berk, B. N. Breizman, and M. Pekker, Plasma Phys. Rep. 23, 778 (1997).

[44] H. L. Berk, B. N. Breizman, J. Candy, M. Pekker, and N. V. Petviashvili, Phys. Plasmas 6, 3102 (1999).

[45] C. Cheng, Phys. Rep. 211, 1 (1992).

[46] N. N. Gorelenkov, Y. Chen, R. B. White, and H. L. Berk, Phys. Plasmas 6, 629 (1999).

[47] R. Hemsworth, H. Decamps, J. Graceffa, B. Schunke, M. Tanaka, M. Dremel, A. Tanga, H. D. Esch, F. Geli, J. Milnes, T. Inoue, D. Marcuzzi, P. Sonato, and P. Zaccaria, Nuclear Fusion 49, 045006 (2009).

[48] M. J. Singh, D. Boilson, A. R. Polevoi, T. Oikawa, 
and R. Mitteau, New Journal of Physics 19, 055004 (2017).

[49] S. Sharapov, B. Alper, H. Berk, D. Borba, B. Breizman, C. Challis, I. Classen, E. Edlund, J. Eriksson, A. Fasoli, E. Fredrickson, G. Fu, M. Garcia-Munoz, T. Gassner, K. Ghantous, V. Goloborodko, N. Gorelenkov, M. Gryaznevich, S. Hacquin, W. Heidbrink, C. Hellesen, V. Kiptily, G. Kramer, P. Lauber, M. Lilley, M. Lisak, F. Nabais, R. Nazikian, R. Nyquist, M. Osakabe, C. P. von Thun, S. Pinches, M. Podesta, M. Porkolab, K. Shinohara, K. Schoepf, Y. Todo, K. Toi, M. V. Zeeland, I. Voitsekhovich, R. White, V. Yavorskij, and ITPA EP TG and JET-EFDA Contributors, Nuclear Fusion 53, 104022 (2013).

[50] P. Lauber, Plasma Physics and Controlled Fusion 57, 054011 (2015).

[51] S. D. Pinches, I. T. Chapman, P. W. Lauber, H. J. C. Oliver, S. E. Sharapov, K. Shinohara, and K. Tani, Physics of Plasmas 22, 021807 (2015), https://doi.org/10.1063/1.4908551.

[52] P. Rodrigues, A. Figueiredo, J. Ferreira, R. Coelho, F. Nabais, D. Borba, N. Loureiro, H. Oliver, and S. Sharapov, Nuclear Fusion 55, 083003 (2015).

[53] M. Schneller, P. Lauber, and S. Briguglio, Plasma Physics and Controlled Fusion 58, 014019 (2016).

[54] M. Fitzgerald, S. Sharapov, P. Rodrigues, and D. Borba, Nuclear Fusion 56, 112010 (2016).

[55] A. Figueiredo, P. Rodrigues, D. Borba, R. Coelho, L. Fazendeiro, J. Ferreira, N. Loureiro, F. Nabais, S. Pinches, A. Polevoi, and S. Sharapov, Nuclear Fusion 56, 076007 (2016).

[56] P. Rodrigues, A. Figueiredo, D. Borba, R. Coelho, L. Fazendeiro, J. Ferreira, N. Loureiro, F. Nabais, S. Pinches, A. Polevoi, and S. Sharapov, Nuclear Fusion 56, 112006 (2016).

[57] N. N. Gorelenkov, H. L. Berk, R. V. Budny, C. E. Kessel, G. J. Kramer, D. McCune, J. Manickam, R. Nazikian, and A. Polevoi, The linear stability properties of medium- to high-n TAEs in ITER,
Tech. Rep. (Princeton Plasma Physics Laboratory, DOE OFES Theory Joule Milestone FY2007, Preprint 4287, 2008).

[58] N. Gorelenkov, H. Berk, and R. Budny, Nuclear Fusion 45, 226 (2005).

[59] M. V. Zeeland, N. Gorelenkov, W. Heidbrink, G. Kramer, D. Spong, M. Austin, R. Fisher, M. G. Muñoz, M. Gorelenkova, N. Luhmann, M. Murakami, R. Nazikian, D. Pace, J. Park, B. Tobias, and R. White, Nuclear Fusion 52, 094023 (2012).

[60] G. Vlad, S. Briguglio, G. Fogaccia, F. Zonca, and M. Schneider, Nuclear Fusion 46, 1 (2006).

[61] M. Y. Isaev, S. Y. Medvedev, and W. A. Cooper, Plasma Physics Reports 43, 109 (2017).

[62] Y. Todo and A. Bierwage, Plasma and Fusion Research 9, 3403068 (2014).

[63] H. Berk, B. Breizman, J. Fitzpatrick, and H. Wong, Nucl. Fusion 35, 1661 (1995).

[64] N. N. Gorelenkov, V. N. Duarte, M. Podesta, and H. L. Berk, Nuclear Fusion (submitted) (2018).

[65] G. Meng, N. N. Gorelenkov, V. N. Duarte, H. L. Berk, R. B. White, and X. Wang, Nuclear Fusion (2018), 10.1088/1741-4326/aaa918.

[66] R. Budny, Nuclear Fusion 49, 085008 (2009).

[67] A. C. C. Sips, for the Steady State Operation, and the Transport Physics topical groups of the International Tokamak Physics Activity, Plasma Physics and Controlled Fusion 47, A19 (2005).

[68] M. Albergante, J. P. Graves, A. Fasoli, F. Jenko, and T. Dannert, Physics of Plasmas 16, 112301 (2009).

[69] M. Albergante, J. Graves, A. Fasoli, and X. Lapillonne, Nuclear Fusion 50, 084013 (2010).

[70] M. Albergante, J. P. Graves, A. Fasoli, M. Jucker, X. Lapillonne, and W. A. Cooper, Plasma Physics and Controlled Fusion 53, 054002 (2011).

[71] F. Jenko, W. Dorland, M. Kotschenreuther, and B. N. Rogers, Physics of Plasmas 7, 1904 (2000), https://doi.org/10.1063/1.874014. 